\title{
Effects of atomic interactions on quantum accelerator modes
}

\author{
Laura Rebuzzini, ${ }^{1,2, *}$ Roberto Artuso, ${ }^{1,3,4}$ Shmuel Fishman, ${ }^{5}$ and Italo Guarneri ${ }^{1,2,3}$ \\ ${ }^{1}$ Center for Nonlinear and Complex Systems and Dipartimento di Fisica e Matematica, Università dell'Insubria, Via Valleggio 11, \\ 22100 Como, Italy \\ ${ }^{2}$ Istituto Nazionale di Fisica Nucleare, Sezione di Pavia, Via Ugo Bassi 6, 27100 Pavia, Italy \\ ${ }^{3}$ Istituto Nazionale di Fisica della Materia, Unità di Como, Via Valleggio 11, 22100 Como, Italy \\ ${ }^{4}$ Istituto Nazionale di Fisica Nucleare, Sezione di Milano, Via Celoria 16, 20133 Milano, Italy \\ ${ }^{5}$ Physics Department, Technion, Haifa 32000, Israel \\ (Received 11 April 2007; published 26 September 2007)
}

\begin{abstract}
We consider the influence of the inclusion of interatomic interactions on the $\delta$-kicked accelerator model. Our analysis concerns in particular quantum accelerator modes, namely quantum ballistic transport near quantal resonances. The atomic interaction is modeled by a Gross-Pitaevskii cubic nonlinearity, and we address both attractive (focusing) and repulsive (defocusing) cases. The most remarkable effect is enhancement or damping of the accelerator modes, depending on the sign of the nonlinear parameter. We provide arguments showing that the effect persists beyond mean-field description, and lies within the experimentally accessible parameter range.
\end{abstract}

DOI: 10.1103/PhysRevA.76.031603

PACS number(s): 03.75.-b, 05.45.Mt, 42.50.Vk

Quantum accelerator modes (QAMs) are a manifestation of a novel type of quantum ballistic transport (in momentum) that has been recently observed in cold atom optics [1]. In these experiments, ensembles of about $10^{7}$ cold alkali-metal atoms are cooled in a magnetic-optical trap to a temperature of a few microkelvin. After releasing the cloud, the atoms are subjected to the joint action of the gravity acceleration and a pulsed potential periodic in space, generated by a standing electromagnetic wave, far detuned from any atomic transitions. The external optical potential is switched on periodically in time and the period is much longer than the duration of each pulse. For values of the pulse period near to a resonant integer multiple of half of a characteristic time $T_{B}$ (the Talbot time [2]), typical of the kind of atoms used, a considerable fraction of the atoms undergo a constant acceleration with respect to the main cloud, which falls freely under gravity and spreads diffusively.

The noninteracting model is a variant of the well-known quantum kicked rotor (KR) [3], in which the effects of a static force, produced by the Earth's gravitational field, are taken into account. The linear potential term breaks invariance of the KR Hamiltonian under space translations. Such an invariance may be recovered by moving to a temporal gauge, where momentum is measured with respect to the free fall: this transformation eliminates the linear term and the Hamiltonian, expressed in dimensionless units, reads

$$
\hat{H}\left(t^{\prime}\right)=\frac{1}{2}\left(\hat{p}+g t^{\prime}\right)^{2}+k \cos (\hat{x}) \sum_{t=-\infty}^{t=+\infty} \delta\left(t^{\prime}-t \tau\right) .
$$

where $\hat{p}$ and $\hat{x}$ are the momentum and position operator, $k$ and $\tau$ are the strength and the temporal period of the external kicking potential, and $g$ is the gravity acceleration. The relationship between the rescaled parameters and the physical

\footnotetext{
*laura.rebuzzini@uninsubria.it
}

ones, denoted by primes, is $k=k^{\prime} / \hbar, \quad \tau=\hbar \tau^{\prime} G^{2} / M$ $=4 \pi \tau^{\prime} / T_{B}, \eta=M g^{\prime} \tau^{\prime} / \hbar G$, and $g=\eta / \tau$, where $\eta$ is the momentum gain over one period, $G$ is twice the angular wave number of the standing wave of the driving potential, and $M$ is the mass of the atom. All the quantities plotted in figures are dimensionless.

Symmetry recovery allows one to decompose the wave packet into a bundle of independent rotors (whose space coordinate is topologically an angle): this Bloch-Wannier fibration plays an important role in the theory of QAMs [4].

QAMs appear when the time gap between kicks approaches a principal quantum resonance, i.e., $\tau=2 \pi l+\epsilon$, with $l$ integer and $|\epsilon|$ small. The key theoretical step is that in this case the quantum propagator may be viewed as the quantization of a classical map, with $|\epsilon|$ playing the role of an effective Planck's constant [4]: QAMs are in correspondence with stable periodic orbits of such pseudoclassical areapreserving map. We refer the reader to the original papers for a full account of the theory, we just mention a few remarkable points: stable periodic orbits are labeled by their action winding number $w=j / q$, which determines the acceleration of the QAM with respect to the center-of-mass distribution

$$
a=\frac{2 \pi}{|\epsilon|} \frac{j}{q}-\frac{\tau \eta}{\epsilon} .
$$

The modes are sensitive to the quasimomentum (Bloch index induced by spatial periodicity), being enhanced at specific, predictable values [4]; also the size of the elliptic island around the pseudoclassical stable orbit plays an important role (if the size is small compared to $|\epsilon|$ the mode is not significant [4]).

We consider here the role of atomic interactions in such a system; namely evolution is determined by a nonlinear Schrödinger equation with a cubic nonlinearity,

$$
i \dot{\psi}\left(x, t^{\prime}\right)=\left[\hat{H}\left(t^{\prime}\right)+u\left|\psi\left(x, t^{\prime}\right)\right|^{2}\right] \psi\left(x, t^{\prime}\right),
$$

where $u$ is the rescaled nonlinear parameter, whose sign describes an attractive (negative) or repulsive (positive) atomic 


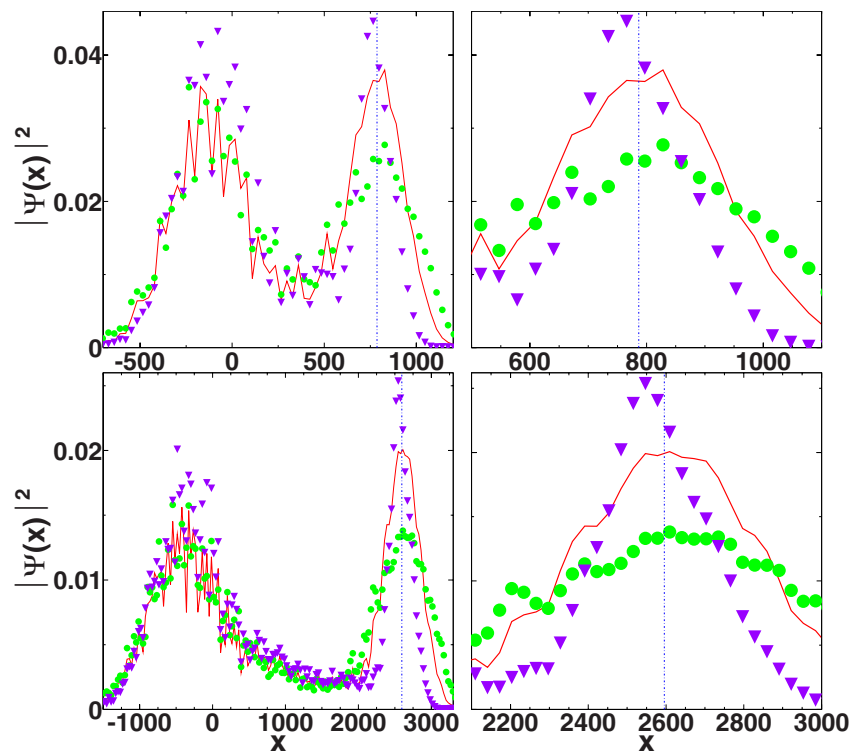

FIG. 1. (Color online) The probability distribution at times $t=25$ (first row) and 45 (second row). (Red) Line: linear case $(u=0)$, (purple) triangles and (green) circles: focusing and defocusing nonlinearity $(u=\mp 1.25)$. In the right-hand column enlargements of mode are shown; the position of the mode, predicted by Eq. (2) is marked by the (blue) vertical dotted line.

interaction. We will come back to its connection with physical units in the end of the paper. The condensate wave function is normalized to unity. The dynamics does not only acquire in this way a qualitatively different form, but, due to the nonlinear term, Bloch decomposition into independent rotors breaks down. The main scope here will be to numerically scrutinize how QAMs are still present in the modified system, and explore how nonlinearity modifies their features. In the end we will briefly comment upon some stability issues, by showing that a more refined description, including loss of thermalized particles, does not destroy the scenario we obtain from a mean-field description.

Our analysis will be restricted to QAMs corresponding to fixed points of period $q=1$ of the pseudoclassical map; the numerical analysis of nonlinear evolution has been performed by using standard time-splitting spectral methods [5]. There are several physical parameters characterizing the system: $g, \tau, k$, and $u$. Here we mainly address the role of nonlinearity $u$ : we fix $k=1.4, l=1, \epsilon=-1, \tau \eta \simeq 0.4173$, and choose as the initial state a symmetric coherent state centered in the stable fixed point of the pseudoclassical map $\left(x_{0} \simeq 0.3027, p_{0}=0\right)$, whose corresponding winding number is zero.

A quite remarkable feature appears when we compare results for opposite nonlinearity signs (keeping the strength $|u|$ fixed), see Fig. 1. As in the linear system, the wave packet splits into two well-separated components: the accelerator mode [whose acceleration is still compatible with Eq. (2)] and the remaining part, which moves under two competitive contributions, the free fall in the gravitation field, and the recoil against the accelerating part. Note that for the present choice of the parameters, the former contribution is negligible compared to the second.
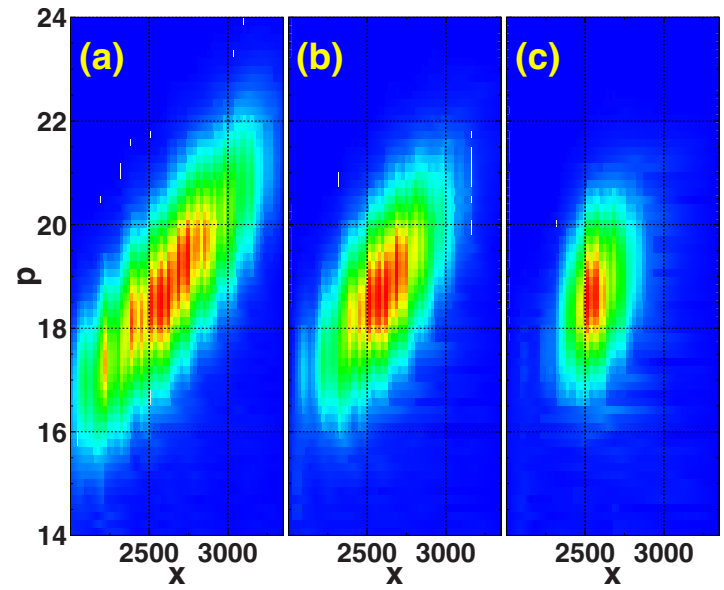

FIG. 2. (Color online) The Husimi function of the QAM at time $t=45$, in the repulsive (a), linear (b), and attractive case (c).

We remark on some features that are common to what we observed for a choice of other parameter values: the distribution around the accelerator mode is more peaked and narrower in the presence of attractive nonlinearity; the opposite happens in the case of a repulsive interaction. This can also be appreciated from a Husimi representation of the modes, providing the probability density for observing the accelerated portion of the atoms in a minimum-uncertainty state, centered at a specified phase space point (see Fig. 2).

While for repulsive interactions the spreading of the distribution, together with peak damping, seems to depend monotonically on the nonlinearity strength, the attractive case exhibits more complicated features (see Fig. 3). Enhancement of the accelerator mode is only observed for small nonlinearities, while a striking feature appears at larger values of $|u|$, namely the accelerator mode is suppressed [see Fig. 4(a)]. The intuitive explanation of this result is that strong focusing nonlinearity opposes to the separation of the wave packet into two parts; indeed, in the case of exact resonance (namely $\tau=2 \pi$ ), the mode is absent, so the whole wave freely falls without splitting and then the maximum height of the wave, plotted vs $u$ as in Fig. 3(a), is found to monotonically increase to the left towards a saturation value. While the behavior shown in Fig. 3 has been observed for a

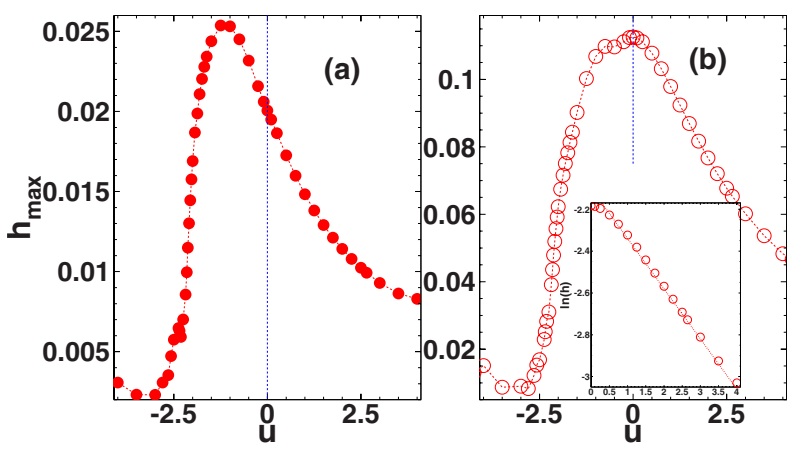

FIG. 3. (Color online) Maximum height reached by the mode at time $t=45$ as a function of $u$ in position (a) and momentum (b) representation. In the inset the exponential decrease of $h_{\max }$ for positive $u$ in shown in a semilogarithmic plot. 


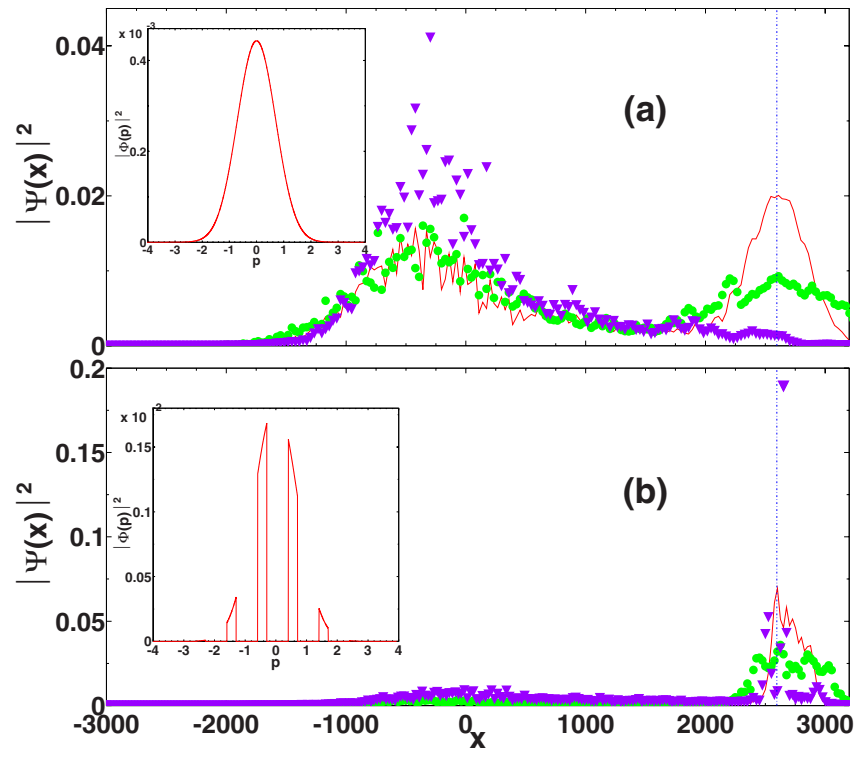

FIG. 4. (Color online) The probability distribution at $t=45$ for strong nonlinearity $(u= \pm 3)$; the initial states (see text) are shown in the insets (line and symbols as in Fig. 1).

variety of other parameter choices, we mention that more complex, strongly fluctuating behavior was sometimes observed at large focusing nonlinearities. In all such cases a bad correspondence between the quantum and the pseudoclassical dynamics was also observed, already in the linear case.

We remark that the mode damping is sensitive to the choice of the initial state, as shown in Fig. 4. While a Gaussian initial wave packet leads to the mentioned QAM suppression, we may tailor a QAM enhancing initial condition as follows: we take the quasimomentum $\beta_{0}$ that in the linear case dominates the mode (here $\beta_{0}=\pi / \tau-\eta / 2 \simeq 0.5551$ [4]) and we drop from the initial Gaussian all components with $\left|\beta-\beta_{0}\right|>0.15$. As quasimomentum is the fractional part of momentum, this leads to the comblike state of Fig. 4(b). Even through quasimomentum is not conserved due to nonlinearity, the QAM is strongly enhanced with respect to the linear case and the recoiling part is almost canceled.

Another way of looking at the nonlinear evolution with techniques that are proper in the linear setting is to consider the distribution function over quasimomenta, defined by

$$
f(\beta, t)=\sum_{n=-\infty}^{+\infty}|\langle n+\beta \mid \psi(t)\rangle|^{2} .
$$

This distribution is stationary under linear evolution, its shape being determined by the choice of the initial state.

We consider the evolution of a Gaussian wave packet [for which the linear $f$ is essentially a constant-the horizontal (red) line of Fig. 5], and probe the effect of nonlinearities of both signs. Typical results are as in Fig. 5: the effect of attractive (repulsive) nonlinearity is to enhance (lower) the distribution around a value $\bar{\beta} \simeq 0.4$. No deviation occurs for quasimomentum $\beta_{0}$ (marked by vertical lines), whose wave function, according to Fig. 4(b), closely follows the linear
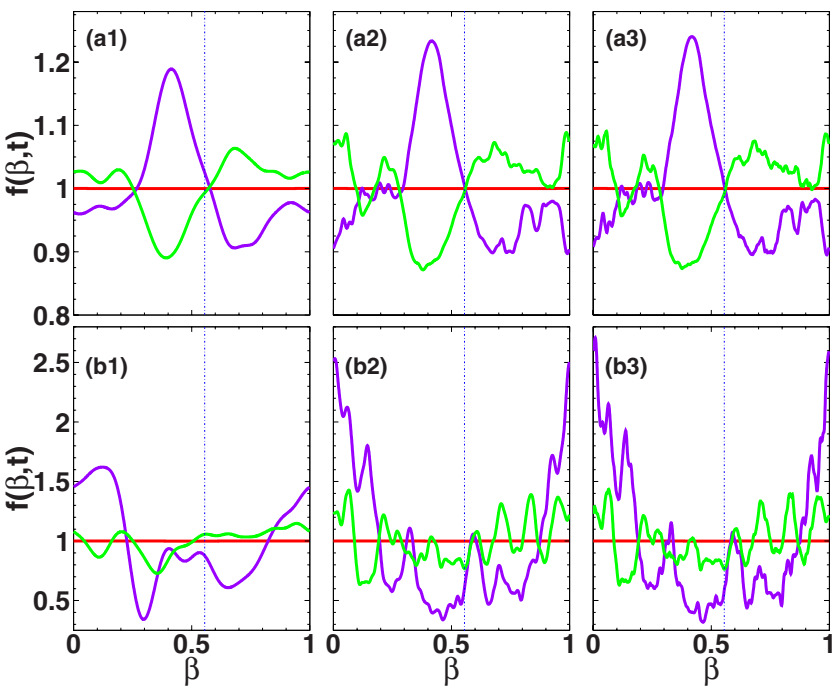

FIG. 5. (Color online) The quasimomentum distribution function (thick line) for $|u|=0.5$ (a) and $|u|=3$ (b) at different times (1) $t=5$, (2) $t=25$, and (3) $t=45$. Purple (dark) and green (light) lines refer to attractive and repulsive interactions.

pseudoclassical island. Again the $\bar{\beta}$ peak of the focusing case is suppressed for large focusing nonlinearities.

To make sure that our findings may be experimentally significant we discuss some stability issues: the first concerns decay properties of the QAMs. It is known that linear modes decay due to quantum tunneling out of pseudoclassical islands [6]: we checked that, on the available time scale, the nonlinear decay behaves in a similar way. In Fig. 6(a) the probability inside the classical island is shown as a function of time for the initial state of Fig. 4(b); it has been calculated integrating the Husimi distribution of each $\beta$-rotor fiber over the island area and summing the contributions of different rotors.

However, in the condensate regime there is another possible mechanism that might completely modify the former picture, namely depletion of the condensate due to proliferation of noncondensed, thermal particles. A standard technique to estimate the growth of the number of thermal particles is provided by the formalism of Castin and Dum [7], which has been employed in similar contexts in [8]. To the
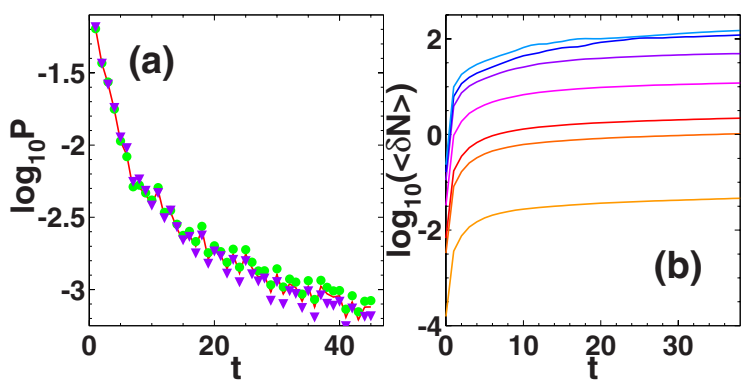

FIG. 6. (Color online) (a) Probability inside the island for $|u|=3$ (symbols and line as in Fig. 1). (b) The mean number of noncondensed particles vs the number of kicks, for $u$ equal to $0.1,0.5$, $0.75,1,2,5,7$, and 10 (starting from below); 12 terms in the sum (5) are considered. 
lowest order in the perturbation expansion and in the limit of zero temperature $T \rightarrow 0$, the number of noncondensed particles is given by

$$
\langle\delta \hat{N}(t)\rangle=\sum_{k}\left\langle v_{k}(t) \mid v_{k}(t)\right\rangle
$$

where $v_{k}(t)$ is one of the mode functions of the system. The modal functions $\left[u_{k}(t), v_{k}(t)\right]$ are pairs of functions that represent the time-dependent coefficients of the decomposition, in terms of annihilation and creation operators, of the equation of motion for the field operator describing the thermal excitations above the condensate. They describe the spatial dependence of these excitations and propagate by modified Bogoliubov equations.

Our findings [see Fig. 6(b)] are consistent with a polynomial growth of noncondensed particles, namely in our parameter region (and within the time scale we typically consider) no exponential instability takes place. This is consistent with recent experimental work [9], where ${ }^{87} \mathrm{Rb}$ atom condensate has been used to explore QAMs. In [9], a condensate of $50000 \mathrm{Rb}$ atoms with repulsive interactions is realized. In the case of a "cigar-shaped" trap, the relationship between the number of atoms in the condensate $N$ and the effective one-dimensional (1D) nonlinear coupling constant $u$ is, in our units, $N=u a_{\perp}^{2} / 2 a_{0}$ [10], where $a_{0}$ is the $3 \mathrm{D}$ scattering length and $a_{\perp} \gg a_{0}$ is the radial extension of the wave function. Using the parameter values of the experiment [9], one finds $N \simeq 10^{5} u$ and so $N \sim 50000$ corresponds to $u \sim 0.5$. Therefore our range of parameters includes the experimental accessible one.

We have investigated effects of atomic interactions, in the form of a cubic nonlinearity, on the problem of quantum accelerator modes. In particular, we have characterized the consequences of both attractive and repulsive interaction; we have also provided evidences that the modes are not strongly unstable when reasonable parameters are chosen.

We thank G.S. Summy for providing us with details of his work. This work has been partially supported by the MIUR-PRIN 2005 project "Transport properties of classical and quantum systems." S.F. acknowledges support by the Israel Science Foundation (ISF), by the US-Israel Binational Science Foundation (BSF), by the Minerva Center of Nonlinear Physics of Complex Systems, and by the Shlomo Kaplansky academic chair. I.G. acknowledges hospitality by the Institute of Theoretical Physics at the Technion where part of this work was done.
[1] M. K. Oberthaler, R. M. Godun, M. B. d'Arcy, G. S. Summy, and K. Burnett, Phys. Rev. Lett. 83, 4447 (1999); R. M. Godun, M. B. d'Arcy, M. K. Oberthaler, G. S. Summy, and K. Burnett, Phys. Rev. A 62, 013411 (2000); S. Schlunk, M. B. d'Arcy, S. A. Gardiner, and G. S. Summy, Phys. Rev. Lett. 90, 124102 (2003); Z.-Y. Ma, M. B. d'Arcy, and S. A. Gardiner, ibid. 93, 164101 (2004).

[2] M. V. Berry and E. Bodenschatz, J. Mod. Opt. 46, 349 (1999).

[3] G. Casati, B. V. Chirikov, F. M. Izrailev, and J. Ford, in Lectures Notes in Physics, edited by G. Casati and J. Ford (Springer, Berlin, 1979), Vol. 93.

[4] S. Fishman, I. Guarneri, and L. Rebuzzini, Phys. Rev. Lett. 89, 084101 (2002); J. Stat. Phys. 110, 911 (2003); I. Guarneri, L.
Rebuzzini, and S. Fishman, Nonlinearity 19, 1141 (2006).

[5] A. D. Bandrauk and H. Shen, J. Phys. A 27, 7147 (1994).

[6] M. Sheinman, S. Fishman, I. Guarneri, and L. Rebuzzini, Phys. Rev. A 73, 052110 (2006).

[7] Y. Castin and R. Dum, Phys. Rev. A 57, 3008 (1998); Phys. Rev. Lett. 79, 3553 (1997).

[8] S. A. Gardiner, D. Jaksch, R. Dum, J. I. Cirac, and P. Zoller, Phys. Rev. A 62, 023612 (2000); C. Zhang, J. Liu, M. G. Raizen, and Q. Niu, Phys. Rev. Lett. 92, 054101 (2004).

[9] G. Behinaein, V. Ramareddy, P. Ahmadi, and G. S. Summy, Phys. Rev. Lett. 97, 244101 (2006).

[10] D. S. Petrov, G. V. Shlyapnikov, and J. T. M. Walraven, Phys. Rev. Lett. 85, 3745 (2000). 